\title{
Detecting differentially methylated loci for Illumina Array methylation data based on human ovarian cancer data
}

\author{
Zhongxue Chen ${ }^{1 *}$, Hanwen Huang ${ }^{2}$, Jianzhong Liu $^{3}$, Hon Keung Tony $\mathrm{Ng}^{4}$, Saralees Nadarajah ${ }^{5}$, Xudong Huang ${ }^{6}$, \\ Youping Deng ${ }^{7}$
}

From The 2011 International Conference on Bioinformatics and Computational Biology (BIOCOMP'11) Las Vegas, NV, USA. 18-21 July 2011

\begin{abstract}
Background: It is well known that DNA methylation, as an epigenetic factor, has an important effect on gene expression and disease development. Detecting differentially methylated loci under different conditions, such as cancer types or treatments, is of great interest in current research as it is important in cancer diagnosis and classification. However, inappropriate testing approaches can result in large false positives and/or false negatives. Appropriate and powerful statistical methods are desirable but very limited in the literature.
\end{abstract}

Results: In this paper, we propose a nonparametric method to detect differentially methylated loci under multiple conditions for Illumina Array Methylation data. We compare the new method with other methods using simulated and real data. Our study shows that the proposed one outperforms other methods considered in this paper.

Conclusions: Due to the unique feature of the Illumina Array Methylation data, commonly used statistical tests will lose power or give misleading results. Therefore, appropriate statistical methods are crucial for this type of data. Powerful statistical approaches remain to be developed.

Availability: $\mathrm{R}$ codes are available upon request.

\section{Background}

It is well known that DNA methylation has important effects on transcriptional regulation, chromosomal stability, genomic imprinting, and $\mathrm{X}$-inactivation [1,2]. It has been also shown to be associated with many human diseases, such as various types of cancer [3-11].

With the advances of BeadArray technology, genomewide high-throughput methylation data can be easily generated by Illumina GoldenGate and Infinium Methylation Assays. After preprocessing steps, such as background correction and normalization, are applied to the raw fluorescent intensities, for each locus, from about 30 replicates in the same array a summarized $\beta$-value is

\footnotetext{
* Correspondence: zc3@indiana.edu

'Department of Epidemiology and Biostatistics, School of Public Health, Indiana University Bloomington, 1025 E. 7th Street, Bloomington, IN 47405, USA

Full list of author information is available at the end of the article
}

generated as follows: $\frac{\max \{M, 0\}}{\max \{M, 0\}+\max \{U, 0\}+100}$, where $M$ is the average signal from a methylated allele while $U$ is that from unmethylated allele. The $\beta$-values are continuous numbers between 0 and 1 , with 0 stands for totally unmethylated and 1 for completely methylated.

It has been shown that the $\beta$-value is rarely normally distributed $[9,12,13]$. Therefore the commonly used $t$-test for case control designs or ANOVA for multiple conditions are not the most powerful approaches when detecting differentially methylated loci. Observing this, Wang has proposed a model-based likelihood ratio test to detect differentially methylated loci for case and control data under the assumption that the $\beta$-value follows a threecomponent normal-uniform distribution [9]. Wang showed that for some situations, their proposed test was better than the simple $\mathrm{t}$-test based on simulation studies. 
However, in their method, Wang did not consider the effect of age, which has been shown highly associated with methylation $[14,15]$. Noticing the importance of age effect, one may use a linear regression with age included as a covariate when analyze methylation data with multiple conditions, such as cancer types. However, the underlying assumption of equal variances may not be satisfied [12]. Therefore the commonly used linear regression method may not be appropriate.

In this paper, we consider methylation data with multiple conditions and propose a nonparametric method which incorporates the age effect in a way through the idea of combining p-values from independent tests $[12,16,17]$. More specifically, we first group subjects into several age groups based on their age; then for each age group, a nonparametric Kruskal-Wallis test is conducted for the given locus and the p-value is recorded. An overall p-value for that locus will be estimated through combining the p-values from all age groups. Using a real methylation data with three conditions and a simulation study, we show that the proposed test is more powerful than other methods, including linear regression.

\section{Method}

\section{Proposed method}

Assume there are $K$ conditions and $G$ age groups. For each age group $g(g=1,2, \ldots, G)$, we apply the nonparametric Kruskal-Wallis test and obtain a p-value $p_{g}^{K W}$, then the overall p-value can be estimated by Fisher test [18]:

$$
p_{K W}=\chi_{d f=2 G}^{2}\left(\chi^{2}>-2 \sum_{g=1}^{G} \log \left(p_{g}^{K W}\right)\right)
$$

\section{Combined ANOVA test}

Similarly, we can use ANOVA to replace KW test for each age group and obtain an overall p-value with $p_{g}^{K W}$ being replaced by the $\mathrm{p}$-value $p_{g}^{\text {ANOVA }}$ from ANOVA test:

$$
p_{\text {ANOVA }}=\chi_{d f=2 G}^{2}\left(\chi^{2}>-2 \sum_{g=1}^{G} \log \left(p_{g}^{A N O V A}\right)\right)
$$

\section{Combined median test}

Another nonparametric test is median test using the following statistic for each age group:

$$
M=4 \sum_{k=1}^{K} \frac{\left(A_{k}-n_{k} / 2\right)^{2}}{n_{k}} \text {, where } A_{k} \text { is the number of }
$$

times that the ranks of individual observations from group $\mathrm{k}$ which excess the median from the pooled data, and $n_{k}$ is the sample size of group $k$. When the sample sizes are large, under the null hypothesis that all samples have the same median, the statistic $M$ has a chi-square distribution with K-1 degrees of freedom. The overall p-value from the combined median test can be calculated:

$$
p_{\text {Median }}=\chi_{d f=2 G}^{2}\left(\chi^{2}>-2 \sum_{g=1}^{G} \log \left(p_{g}^{\text {Median }}\right)\right)
$$

\section{Combined welch test}

We also consider the nonparametric Welch test. For each age group, we have the test statistic [19]:

$$
W=\frac{\sum_{k=1}^{K} w_{k}\left(\bar{x}_{k}-\hat{\mu}\right)^{2} /(K-1)}{1+\left[2(K-2) /\left(K^{2}-2\right)\right] \sum_{k=1}^{K} h_{k}},
$$

where $w_{k}=n_{k} / s_{k^{\prime}}^{2}, \hat{\mu}=\sum_{k=1}^{K} w_{k} \bar{x}_{k} / w, \mathrm{w}=\sum_{k=1}^{K} w_{k}, \mathrm{~h}_{k}=\left(1-w_{k} / w^{2}\right) /\left(n_{k}-1\right)$. Under the null hypothesis, the statistic $\mathrm{W}$ is asymptotically distributed as F-distribution with $K-1$ and $\mathrm{f}=\left(\mathrm{K}^{2}-1\right) /\left(3 \sum_{k=1}^{K} h_{k}\right)$ degrees of freedom. Welch test is an improvement of the Cochran test [20] which usually has inflated type I error rate especially for small sample sizes $[19,21]$. The overall $p$-value from the combined Welch test is:

$$
p_{\text {Welch }}=\chi_{d f=2 G}^{2}\left(\chi^{2}>-2 \sum_{g=1}^{G} \log \left(p_{g}^{\text {Welch }}\right)\right)
$$

\section{Methods for combining p-values}

Besides the Fisher method mentioned above, we also consider Z-test to combine p-values from independent tests. First we calculated the weighted $\mathrm{Z}$ statistic using individual $\mathrm{p}$-values from each age group: $Z=\sum_{g=1}^{G} n_{g} \Phi^{-1}\left(1-p_{g}\right) / \sum_{g=1}^{G} n_{g}^{2}$, where $n_{g}$ is the total sample size in age group $g$ and $\Phi$ is the cumulative distribution function (CDF) of the standard normal distribution. It is easy to see that this statistic has standard normal distribution under the null hypothesis. The overall p-value is calculated by $1-\Phi(Z)$. Note that here we use one-sided test to obtain the overall p-value.

\section{Simulation settings}

To compare each method applied to an individual age group, we simulate $\beta$-value for three treatment groups based on beta distribution with parameters $a$ and $b$, beta $(a, b)$, and truncated normal distribution on $(0,1)$ with parameters $\mu, \sigma^{2}, \mathrm{TN}\left(\mu, \sigma^{2}\right)$. We assume the sample sizes (denoted as $s$ in Tables 1, 2 for the simulation results) for the three treatments are either balanced: $s=30$ for each, or non-balanced: $s=20,30$, and 40. First we compare the 
Table 1 Estimated type I error rates at significance level 0.05 with 10000 replicates.

\begin{tabular}{|c|c|c|c|c|}
\hline Distribution (sample sizes, parameters) & ANOVA & median & Welch & KW \\
\hline Beta $(s=30,30,30, a=1,1,1, b=2,2,2)$ & 0.048 & 0.040 & 0.052 & 0.047 \\
\hline Beta $(s=30,30,30, a=1,1,1, b=10,10,10)$ & 0.052 & 0.044 & 0.053 & 0.051 \\
\hline Beta $(s=30,30,30, a=10,10,10, b=1,1,1)$ & 0.047 & 0.044 & 0.052 & 0.048 \\
\hline Beta $(s=30,30,30, a=10,10,10, b=10,10,10)$ & 0.045 & 0.045 & 0.047 & 0.046 \\
\hline Beta $(s=20,30,40, a=1,1,1, b=2,2,2)$ & 0.053 & 0.052 & 0.050 & 0.053 \\
\hline Beta $(s=20,30,40, a=1,1,1, b=10,10,10)$ & 0.049 & 0.049 & 0.054 & 0.048 \\
\hline Beta $(s=20,30,40, a=10,10,10, b=1,1,1)$ & 0.045 & 0.049 & 0.056 & 0.044 \\
\hline Beta $(s=20,30,40, a=10,10,10, b=10,10,10)$ & 0.050 & 0.051 & 0.043 & 0.052 \\
\hline $\operatorname{TN}\left(s=30,30,30, \mu=0.5,0.5,0.5, \sigma^{2}=0.1,0.1,0.1\right)$ & 0.050 & 0.044 & 0.053 & 0.045 \\
\hline $\mathrm{TN}\left(s=30,30,30, \mu=0.5,0.5,0.5, \sigma^{2}=0.1,0.2,0.3\right)$ & 0.053 & 0.067 & 0.047 & 0.053 \\
\hline $\operatorname{TN}\left(s=20,30,40, \mu=0.5,0.5,0.5, \sigma^{2}=0.1,0.1,0.1\right)$ & 0.050 & 0.052 & 0.052 & 0.049 \\
\hline $\operatorname{TN}\left(s=20,30,40, \mu=0.5,0.5,0.5, \sigma^{2}=0.1,0.2,0.3\right)$ & 0.047 & 0.054 & 0.051 & 0.043 \\
\hline
\end{tabular}

estimated type I error rates with the given significance level of 0.05 under the null hypothesis of no differences among treatment groups. Then we compare the empirical powers from each method under various situations. The empirical power is the proportion of rejected null hypothesis to the number of replicates.

\section{A real data set}

We will use a real methylation data set, the United Kingdom Ovarian Cancer Population Study (UKOPS) [15] with 274 controls, 131 pre-treatment cases, and 135 post treatment cases, to compare the performance of the proposed test with others. Those methylation data were generated by the Illumina Infinium Huamn Methylaytion27 BeadChip and can be downloaded under accession number GSE19711 from the NCBI Gene Expression Omnibus (http://www.ncbi.nlm.nih.gov/geo).

For this data set, there are 27578 loci. After data quality control process, we removed some subjects with BS values less than 4000 or the coverage rates less than $95 \%$. We also separate subjects into 6 age groups
(50-55, 55-60, 60-65, 65-70, 70-75, and 75 and over). Table 3 gives the numbers of subjects in each age by treatment groups. For each locus, we perform the above mentioned approaches.

\section{Results}

Simulation results

Table 1 reports the estimated type I error rates from each method under different conditions. For most of the time, the estimated type I error rates are close to the nominal significance level as expected. Table 2 gives the empirical powers from each method. It can be seen that the non-parametric method of Mood's median test usually has the lowest powers in the simulations. None of the ANOVA, Welch and KW tests is uniformly most powerful. In words, their performances depend on the distributions from which the data are generated. From our simulation study, the KW test is usually as powerful as or more powerful than the ANOVA test. The true distributions of the $\beta$-value may vary from locus to locus; it is impossible to simulate all possible

Table 2 Empirical power at significance level 0.05 with 10000 replicates.

\begin{tabular}{|c|c|c|c|c|}
\hline Distribution (sample sizes, parameters) & ANOVA & median & Welch & KW \\
\hline $\operatorname{Beta}(s=30,30,30, a=5,5,5, b=20,25,30$ & 0.821 & 0.576 & 0.810 & 0.775 \\
\hline $\operatorname{Beta}(s=30,30,30, a=1.5,2,2.5, b=20,20,20$ & 0.650 & 0.504 & 0.648 & 0.710 \\
\hline Beta $(s=30,30,30, a=20,20,20, b=1.5,2,2.5$ & 0.658 & 0.495 & 0.656 & 0.713 \\
\hline $\operatorname{Beta}(s=20,30,40, a=5,5,5, b=20,25,30)$ & 0.792 & 0.546 & 0.740 & 0.735 \\
\hline $\operatorname{Beta}(s=20,30,40, a=1.5,2,2.5, b=20,20,20)$ & 0.599 & 0.479 & 0.634 & 0.670 \\
\hline $\operatorname{Beta}(s=20,30,40, a=20,20,20, b=1.5,2,2.5)$ & 0.607 & 0.475 & 0.637 & 0.665 \\
\hline $\operatorname{TN}\left(s=30,30,30, \mu=0.45,0.5,0.55, \sigma^{2}=0.2\right)$ & 0.383 & 0.240 & 0.378 & 0.362 \\
\hline $\operatorname{TN}\left(s=30,30,30, \mu=0.45,0.5,0.55, \sigma^{2}=0.1,0.2,0.3\right)$ & 0.338 & 0.325 & 0.412 & 0.341 \\
\hline $\operatorname{TN}\left(s=20,30,40, \mu=0.45,0.5,0.55, \sigma^{2}=0.2\right)$ & 0.349 & 0.238 & 0.343 & 0.328 \\
\hline $\operatorname{TN}\left(s=20,30,40, \mu=0.45,0.5,0.55, \sigma^{2}=0.1,0.2,0.3\right)$ & 0.219 & 0.361 & 0.423 & 0.259 \\
\hline
\end{tabular}


Table 3 Number of samples in age group by treatment group used in the paper after removing subjects with bs $<4000$ or coverage rate $<95 \%$ or age $>80$.

\begin{tabular}{lllll}
\hline Age group & control & Pre-treat & Post-treat & Total \\
\hline $50 \_55$ & 14 & 15 & 16 & 45 \\
$55 \_60$ & 61 & 17 & 25 & 103 \\
$60 \_65$ & 64 & 17 & 22 & 103 \\
$65 \_70$ & 35 & 17 & 21 & 73 \\
$70 \_75$ & 63 & 24 & 22 & 109 \\
75_over & 20 & 18 & 9 & 47 \\
Total & 257 & 108 & 115 & 480 \\
\hline
\end{tabular}

distributions. However, based on the observation of the real data, we know that the distributions of the $\beta$-value are far from the normal distribution, under which ANOVA is the best test. Therefore, we prefer nonparametric tests which are more robust.

\section{Results from real data set}

or the real data set, we applied the above mentioned methods to get the overall p-values (either using Fisher or $\mathrm{Z}$ test to combine $\mathrm{p}$-values from individual age groups) for each locus. Then we use various cutoff $\mathrm{p}$ values, $0.001,0.0001,0.00001$, and 0.000001 , to count how many loci have smaller p-values for each method. Table 4 reports the results. We can see that the KW method usually finds more significant loci than other methods. It also shows that the two combining p-value methods, Fisher and $\mathrm{Z}$ test have similar performances, although $\mathrm{Z}$ test usually give a little bit more significant loci expect for the Median test. Figure 1 plots the negative $\log 10 \mathrm{p}$-values from pairs of the methods. It shows that the KW method gives smaller p-values especially when the differences among the three treatment groups are not large (e.g., the negative log10 pvalues between 3 and 6). From Figure 1 we can see that for a given cutoff $\mathrm{p}$-value, most of the loci identified by ANOVA test or Median were also detected by the Welch test; in turn, most of the loci identified by Welch test were also detected by the KW test. This indicates the KW test is more powerful than other methods compared.

\section{Discussion and conclusions}

Due to the unique feature of the $\beta$-value of methylation data, traditional statistical methods, such as linear regression and ANOVA test may not be appropriate. It has been shown that methylation is highly correlated with age; ignoring age effect may cause many false positives and/or false negatives. The effect of age may also not be linear; therefore we need a better way to account for this effect. In this paper, we use p-value combination method to deal with age effect. For each age group, we use nonparametric method to compare the treatment groups. It is important to find powerful and robust nonparametric methods for this sort of data. Although we found that KW method is more powerful than some other nonparametric methods for methylation data, it is desirable to find more powerful tests in this area. Furthermore, we want to point out that there are many other methods can be used to combine p-values [22,23]; it may also be possible to find a more powerful method to combine p-values for Illumina Array Methylation data. However, based on our experiences, Fisher test is more robust and can be used in situations when a small portion of the p-values are very small; while the $\mathrm{Z}$ test is more powerful when the effect sizes are similar (e.g., the p-values don't differ much) for all of the age groups. Finally, although in this paper we use different cutoff p-values to compare the performance of tests, one may want to control the false positive rate. Several multiple comparison methods have been proposed for large scale data set to deal with the situations where the variables (loci) are not independent [24-28]. However, it

Table 4 Number of significant differentially methylated loci detected for given cutoff p-value based on the real data.

\begin{tabular}{|c|c|c|c|c|c|c|c|c|}
\hline \multirow[t]{2}{*}{ Method } & \multicolumn{2}{|c|}{$1 \mathrm{e}-3$} & \multicolumn{2}{|c|}{$1 e-4$} & \multicolumn{2}{|c|}{$1 \mathrm{e}-5$} & \multicolumn{2}{|c|}{$1 e-6$} \\
\hline & Fisher & Z-test & Fisher & Z-test & Fisher & Z-test & Fisher & Z-test \\
\hline ANOVA & 981 & 1079 & 655 & 690 & 479 & 499 & 350 & 375 \\
\hline Median & 906 & 893 & 464 & 449 & 255 & 240 & 143 & 127 \\
\hline Welch & 1096 & 1106 & 640 & 673 & 416 & 424 & 281 & 289 \\
\hline$K-W$ & 1359 & 1340 & 823 & 859 & 551 & 590 & 381 & 401 \\
\hline
\end{tabular}




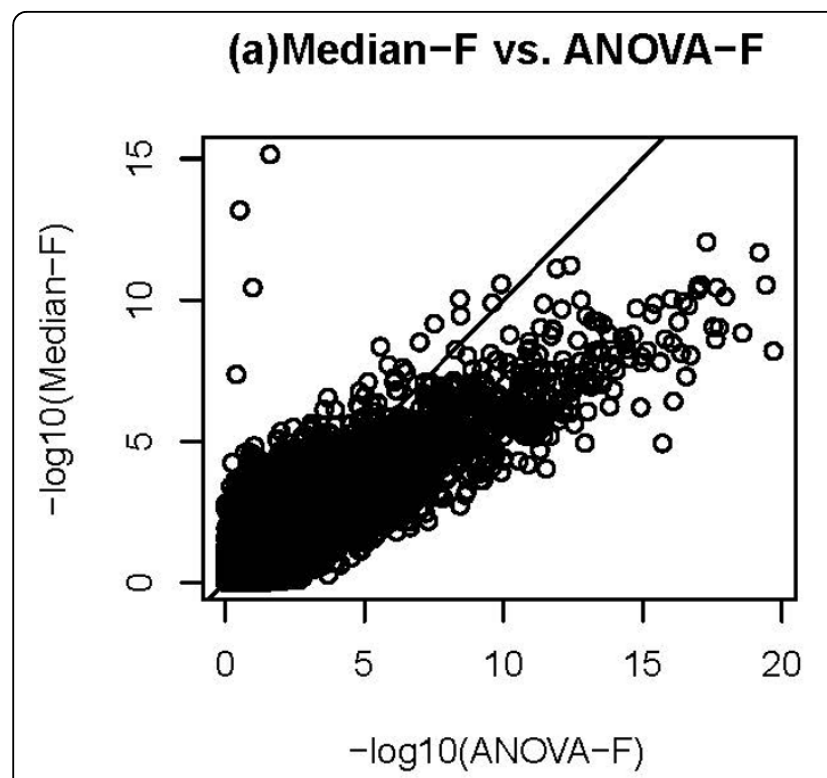

(c)KW-F vs. ANOVA-F

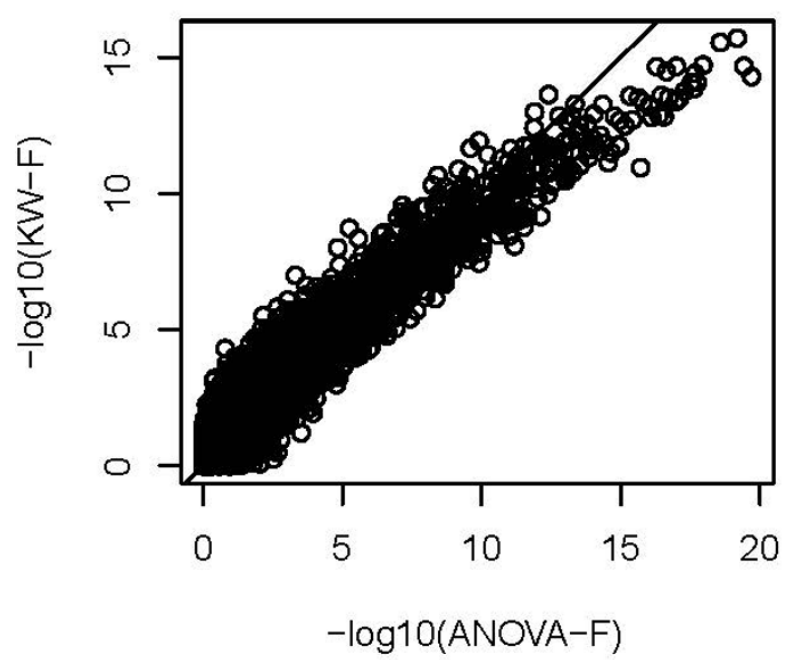

(b)Welch-F vs. ANOVA-F

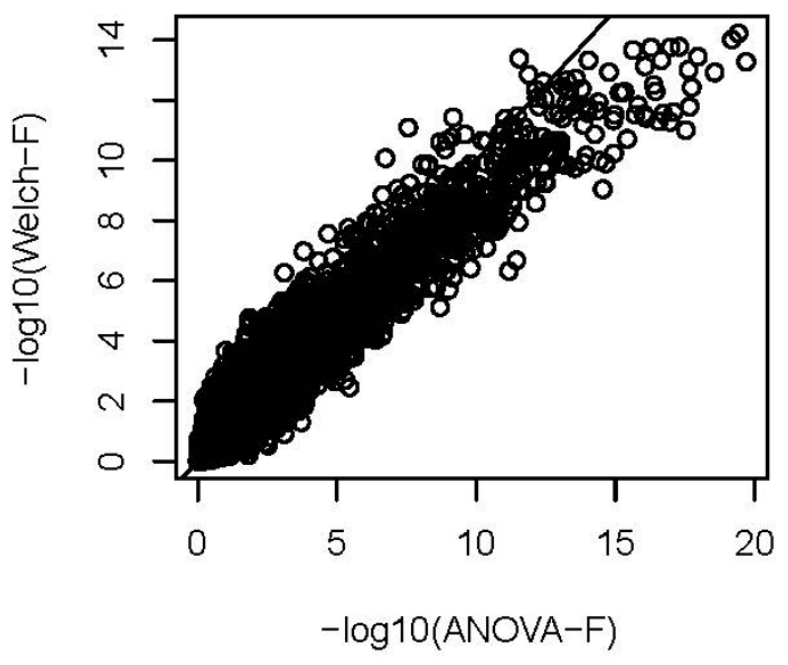

(d)KW(Z vs.Fisher)

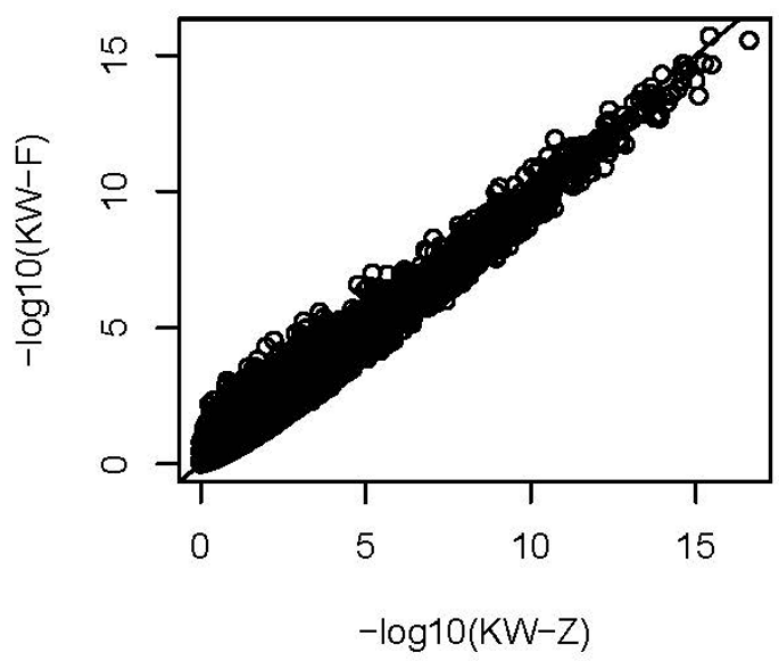

Figure 1 Negative log10(p-value) from pair of methods. Negative log10 p-values from pair of methods. (a) Combined ANOVA test vs. combined median test both using Fisher methods to combine p-values. (b) Combined ANOVA test vs. combined Welch test both using Fisher methods to combine p-values. (c) Combined ANOVA test vs. combined Kruskal-Willas test both using Fisher methods to combine p-values. (d) Combined KW test using Z test vs. combined KW test using Fisher to combine p-values.

remains to study which approach is more appropriate for the methylation data.

\section{Authors' contributions}

ZC devised the basic idea of the new method and drafted the manuscript; $\mathrm{HH}, \mathrm{J}$ participated in study design data analysis; HKTN, SN, XH and YD assisted the study and co-wrote the manuscript. All authors read and approve the final manuscript.

\section{Competing interests}

The authors declare that they have no competing interests.
Acknowledgements

This article has been published as part of BMC Medical Genomics Volume 6 Supplement 1, 2013: Proceedings of the 2011 International Conference on Bioinformatics and Computational Biology (BIOCOMP'11). The full contents of the supplement are available online at http://www.biomedcentral.com/ bmcmedgenomics/supplements/6/S1. Publication of this supplement has been supported by the International Society of Intelligent Biological Medicine.

\section{Author details}

'Department of Epidemiology and Biostatistics, School of Public Health, Indiana University Bloomington, 1025 E. 7th Street, Bloomington, IN 47405, 
USA. ${ }^{2}$ Center for Clinical and Translational Sciences, The University of Texas Health Science Center at Houston, Houston, TX 77030, USA. ${ }^{3}$ Chem 21 Group, Inc, 1780 Wilson Drive, Lake Forest, IL 60045, USA. ${ }^{4}$ Department of Statistical Science, Southern Methodist University, Dallas, TX 75275, USA. ${ }^{5}$ School of Mathematics, University of Manchester, Manchester, M13 9PL, UK.

${ }^{6}$ Neurochemistry Laboratory, Department of Psychiatry, Massachusetts General Hospital and Harvard Medical School, Charlestown, MA 02129, USA ${ }^{7}$ Rush University Cancer Center, Department of Internal Medicine and Biochemistry, Rush University Medical Center, Chicago, IL 60612, USA.

Published: 23 January 2013

\section{References}

1. Kuan PF, Wang S, Zhou X, Chu H: A statistical framework for Illumina DNA methylation arrays. Bioinformatics 2010, 26(22):2849.

2. Rakyan VK, Down TA, Thorne NP, Flicek P, Kulesha E, Gräf S, Tomazou EM, Bäckdahl L, Johnson N, Herberth M: An integrated resource for genomewide identification and analysis of human tissue-specific differentially methylated regions (tDMRs). Genome research 2008, 18(9):1518-1529.

3. Baylin SB, Ohm JE: Epigenetic gene silencing in cancer-a mechanism for early oncogenic pathway addiction? Nature Reviews Cancer 2006, 6(2):107-116.

4. Feinberg AP, Tycko B: The history of cancer epigenetics. Nature Reviews Cancer 2004, 4(2):143-153.

5. Jabbari K, Bernardi G: Cytosine methylation and CpG, TpG (CpA) and TpA frequencies. Gene 2004, 333:143-149.

6. Jones PA, Baylin SB: The fundamental role of epigenetic events in cancer. Nature Reviews Genetics 2002, 3(6):415-428.

7. Kulis M, Esteller M: DNA methylation and cancer. Adv Genet 2010, 70:27-56

8. Laird PW: Principles and challenges of genome-wide DNA methylation analysis. Nature Reviews Genetics 2010, 11(3):191-203.

9. Wang S: Method to detect differentially methylated loci with casecontrol designs using Illumina arrays. Genetic Epidemiology 2011 35(December):686-694.

10. Xu GL, Bestor TH, Bourc'his D, Hsieh CL, Tommerup N, Bugge M, Hulten M, Qu X, Russo JJ, Viegas-Péquignot E: Chromosome instability and immunodeficiency syndrome caused by mutations in a DNA methyltransferase gene. Nature 1999, 402(6758):187-191.

11. Hansen KD, Timp W, Bravo HC, Sabunciyan S, Langmead B, McDonald OG, Wen B, Wu H, Liu Y, Diep D: Increased methylation variation in epigenetic domains across cancer types. Nature Genetics 2011, 43(8):768-775.

12. Chen Z, Liu Q, Nadarajah S: A new statistical approach to detecting differentially methylated loci for case control Illumina array methylation data. Bioinformatics 2012, 28(8):1109-1113.

13. Huang $H$, Chen $Z$ : Age adjusted nonparametric detection of differential DNA methylation with case-control designs. Unpublished manuscript .

14. Christensen BC, Houseman EA, Marsit CJ, Zheng S, Wrensch MR, Wiemels $J$, Nelson HH, Karagas MR, Padbury JF, Bueno R: Aging and environmental exposures alter tissue-specific DNA methylation dependent upon CpG island context. PLoS genetics 2009, 5(8):e1000602.

15. Teschendorff AE, Menon U, Gentry-Maharaj A, Ramus SJ, Weisenberger DJ, Shen H, Campan M, Noushmehr H, Bell CG, Maxwell AP: Age-dependent DNA methylation of genes that are suppressed in stem cells is a hallmark of cancer. Genome research 2010, 20(4):440-446.

16. Chen $\mathrm{Z}, \mathrm{Ng} H \mathrm{HKT}$ : A robust method for testing association in genomewide association studies. Human Heredity 2012, 73(1):26-34.

17. Chen Z: A new association test based on Chi-square partition for casecontrol GWA studies. Genetic Epidemiology 2011, , 35: 658-658.

18. Fisher RA: Statistical methods for research workers. Edinburgh: Oliver and Boyd 1932.

19. Welch B: On the comparison of several mean values: An alternative approach. Biometrika 1951, 38(3/4):330-336.

20. Cochran WG: Problems arising in the analysis of a series of similar experiments. Journal of Royal Statistical Society, Series C: Applied Statistics 1937, 4:102-118.

21. Chen Z, Ng HKT, Nadarajah S: A note on Cochran test for homogeneity in one-way ANOVA and meta-analysis. Statistical Papers 2012, 1-10.

22. Chen $Z$ : Is the weighted $z$-test the best method for combining probabilities from independent tests? Journal of Evolutionary Biology 2011, 24(4):926-930.
23. Chen Z, Nadarajah S: Comments on 'Choosing an optimal method to combine p-values' by Sungho Won, Nathan Morris, Qing Lu and Robert C. Elston, Statistics in Medicine 2009; 28: 1537-1553. Statistics in Medicine 2011, 30(24):2959-2961.

24. Dudbridge F, Gusnanto A: Estimation of significance thresholds for genomewide association scans. Genet Epidemiol 2008, 32(3):227-234.

25. Gao X, Starmer J, Martin ER: A multiple testing correction method for genetic association studies using correlated single nucleotide polymorphisms. Genet Epidemiol 2008, 32(4):361-369.

26. Moskvina $V$, Schmidt KM: On multiple-testing correction in genome-wide association studies. Genet Epidemiol 2008, 32(6):567-573.

27. Pe'er I, Yelensky R, Altshuler D, Daly MJ: Estimation of the multiple testing burden for genomewide association studies of nearly all common variants. Genet Epidemiol 2008, 32(4):381-385.

28. Chen Z, Liu Q: A new approach to account for the correlations among single nucleotide polymorphisms in genome-wide association studies. Human Heredity 2011, 72(1):1-9.

doi:10.1186/1755-8794-6-S1-S9

Cite this article as: Chen et al:: Detecting differentially methylated loci for Illumina Array methylation data based on human ovarian cancer data. BMC Medical Genomics 2013 6(Suppl 1):S9.

\section{Submit your next manuscript to BioMed Central and take full advantage of:}

- Convenient online submission

- Thorough peer review

- No space constraints or color figure charges

- Immediate publication on acceptance

- Inclusion in PubMed, CAS, Scopus and Google Scholar

- Research which is freely available for redistribution 